\title{
Capacity and Reliability Function per Fourth Moment Cost for WSSUS Fading Channels
}

\author{
Bruce Hajek ${ }^{1}$ and Vijay G. Subramanian \\ Coordinated Science Laboratory and the ECE Dept., Univ. of Illinois, Urbana, IL 61801. \\ Email:\{b-hajek,vgsubram\} @uiuc.edu.
}

\begin{abstract}
This paper addresses the capacity of wide sense stationary uncorrelated scattering (WSSUS) fading channels. Associated with a given input signal we define a quantity called the "fourthegy" of the signal, relative to a given WUSSUS channel. The name is inspired by the fact that the measure is fourth order in the input signal amplitude. The fourthegy depends on the signal through its ambiguity function, and on the channel through a simple channel response function. The maximum possible mutual information for the channel per unit fourthegy is found.

Roughly speaking, the fourthegy is a sum over time and frequency bins of the local signal energy squared. The fourthegy-to-energy ratio of directsequence spread spectrum signals is inversely propoportional to bandwidth. Therefore, for such signals, the capacity per unit energy (or the capacity per unit time for fixed power) tends to zero as the bandwidth increases. This does not happen to signals that are more bursty in time-frequency space, such as frequency-hopped signals or M-ary Frequency Shift Keyed signals. A similar result was found by Gallager and Medard for a less conventional channel model. Numerical evaluation of the bound shows it to be informative only for rather large bandwidths.
\end{abstract}

\section{Wireless Channel Models}

A time-varying linear model of a wireless channel is adopted. The output $y(t)$ of the channel is given by

$$
y(t)=\int h(t, \tau) u(t-\tau) d \tau+n(t),
$$

where $u(t)$ is the input, $h(t, \tau)$ is the time-varying channel transfer function, and $n(t)$ is white Gaussian noise. It is assumed that $h(t, \tau)$ for fixed $\tau$ is a wide-sense stationary (WSS) process, that $h(t, \tau)$ is a Gaussian random process. Uncorrelated scattering (US) is also assumed, meaning that the variables $h(t, \tau)$ for different values of $\tau$ are uncorrelated.

\section{Capacity and Reliability Function Per Unit Cost}

Gallager[2] in his seminal work discussed energy limited channels, i.e., channels where the energy per degree of freedom is very small. Restricting the input to binary signals he computed the reliability function [1] per unit cost. The cost could be the energy or could be something else, but it is assumed that some input $\mathbf{0}$ has zero cost. Gallager showed that the reliability function per unit cost is given by,

$$
\hat{E}_{r}(\hat{R})=\max _{0 \leq \rho \leq 1}\left(\sup _{u: \operatorname{cost}(u) \neq 0} \frac{\left.\left(-(1+\rho) \log E\left[\Lambda^{1 /(1+\rho)}\right] U=0\right]\right)}{\cos (u)}\right)-\rho \hat{R},
$$

${ }^{1}$ This work was supported by a Motorola Fellowship, by the US Army Research Office under Grant DAAH05-95-1-0246, and by the National Science Foundation under contract NSF NCR 93-14253. where $\operatorname{cost}(u)$ is the cost associated with input $u$, and $\Lambda=$ $d P_{Y \mid U=u} / d P_{Y \mid U=0}$ is the likelihood ratio of the input $u$ with respect to the 0 input. Verdú[7] considered capacity per unit energy cost and showed that

$$
C_{\text {cost }}=\sup _{x: \operatorname{cost}(x) \neq 0} \frac{D\left(P_{Y \mid X=x} \| P_{Y \mid X=0}\right)}{\operatorname{cost}(x)}
$$

where $D(\cdot \| \cdot)$ is the Kullback-Liebler distance between measures.

\section{Capacity and Reliability Function Calculations}

Consider signaling over a time interval of duration $T$, with $T$ much larger than the coherence time and maximum delay spread of the channel, so that the capacity per unit time (or per unit energy or per other units) over the time interval is representative of the corresponding long-term capacity. Let a WUSSUS channel be given by (1) such that $h$ is complexvalued and Gaussian with mean zero and autocorrelation function $R_{H}(t-s, \tau) \delta(\tau-v)=E\left[h(t, \tau) h^{*}(s, v)\right]$, and suppose $n(t)$ is white, complex-valued Gaussian noise with one-sided power spectral density $\sigma^{2}$. We also constrain the input waveforms to have finite energy.

Equation (1) can be written in the following manner

$$
y(t)=s_{\text {out }}(t)+n(t), \quad 0 \leq t \leq T,
$$

where, given $u, s_{\text {out }}(t)$ is a complex-valued, zero mean Gaussian random process independent of $n(t)$ with covariance function given by $\Sigma(s, t)=E\left[s_{\text {out }}(s) s_{\text {out }}^{*}(t)\right]$. Let $\left\{\lambda_{i}\right\}_{i=0}^{\infty}$ be the eigenvalues of the covariance operator, $\Sigma$. Mercer's theorem using the Karhunen-Loeve expansion yields a simple expression for $D\left(P_{Y \mid X=x} \| P_{Y \mid X=0}\right)$ and $\Lambda$ in terms of the eigenvalues.

\section{Capacity per unit fourth-moment cost}

Define the fourthegy of an input signal $u$ by $J_{C}(u)=$ $\sum_{i=0}^{\infty} \lambda_{i}^{2} .{ }^{1}$ Another representation for the fourthegy $J_{C}(u)$ is

$$
J_{C}(u)=\int_{\nu} \int_{\tau}|\chi(\nu, \tau)|^{2} \psi_{H}(\nu, \tau) d \tau d \nu
$$

where $\chi(\tau, \nu)$ is the symmetric ambiguity function [5] of the signal $u(t)$ which is defined as follows

$$
\chi(\nu, \tau)=\int_{-\infty}^{\infty} u(t+\tau / 2) u^{*}(t-\tau / 2) e^{-j 2 \pi \nu t} d t
$$

'Admittedly the name "fourthegy" lacks luster, but we feel that a postive sounding name, like energy, is needed, rather than a negative sounding name, like fourth moment cost. Our bounds show that a certain amount of fourthegy is needed per bit for diffuse WUSSUS fading channels, just as a certain amount of energy per bit is needed for either WUSSUS or additive Gaussian channels. 
and where the channel response function $\psi_{H}(\tau, \nu)$ is given by

$$
\psi_{H}(\nu, \tau)=\int_{f} \int_{t} S_{H}(f, t) S_{H}(f+\nu, t+\tau) d t d f .
$$

Note that the fourthegy $J_{C}(u)$ is fourth-order in the signal input, and that $J_{C}(u)$ captures both time and frequency aspects of the signal. It can be shown that $J_{C}(u) \leq G_{H}^{2} \int|u(t)|^{4} d t$ and similarly that $J_{C}(u) \leq G_{H}^{2} \int|U(f)|^{4} d f$ where $U(f)$ is the Fourier transform of $u(t)$. We can, thereafter, show the following

$$
\sup _{u \neq 0} \frac{D(u)}{J_{C}(u)}=\frac{1}{2 \sigma^{4}}
$$

Considering $J_{C}(u)$ as a cost function and applying the result of Verdú [7] we can derive the capacity of the channel per unit fourthegy.

Theorem III.1 The capacity per unit fourthegy, $C_{J}$, of the WSSUS fading channel is given by

$$
C_{J}=\frac{1}{2 \sigma^{4}}
$$

As a consequence we have for any input random process $U$,

$$
I(U ; Y) \leq \frac{1}{2 \sigma^{4}} E\left[J_{C}(U)\right]
$$

where $Y$ is the output random process and the expectation is carried out with respect to the measure of $U$.

A point that we must emphasize here is that Kennedy [4] defines the number of effective diversity paths, $D$ to be the reciprocal of $J_{C}(u)$. In [4] $u$ is the M-ary FSK waveform while here it is the on signal for on-off keying. Thus, $D$ increasing without bound implies that $J_{C}(u)$ decreases to zero and the result of the error exponent going to zero in [4] is mirrored by the mutual information between the input and the output going to zero.

Before going to the next topic we state an important property of ambiguity functions. The volume invariance property [5] states that

$$
\iint|\chi(\nu, \tau)|^{2} d \tau d \nu=\chi(0,0)^{2}=\left(\int|u(t)|^{2} d t\right)^{2}=E(u)^{2} .
$$

Note also that $|\chi(\nu, \tau)| \leq \chi(0,0)=E(u)$.

\section{Reliability function per unit fourthegy}

We can show that $\tilde{E}_{r}(\tilde{R})$ is given as follows

$$
\tilde{E}_{r}(\tilde{R})=\frac{1}{2 \sigma^{4}} \beta\left(2 \sigma^{4} \tilde{R}\right),
$$

where

$$
\beta(r)=\left\{\begin{array}{ll}
\frac{1}{2}-r & 0 \leq r \leq \frac{1}{4} \\
(1-\sqrt{r})^{2} & \frac{1}{4} \leq r \leq 1
\end{array} .\right.
$$

It is interesting to note that we get an expression for the reliability function which does not depend on the channel except through $\sigma^{2}$. Also, the shape of the reliability function is exactly that of the reliability function per unit energy for an infinite bandwidth AWGN channel [1, p. 381].

\section{APplications TO DS-CDMA CAPACITY}

We concentrate on DS-CDMA signals of the type

$$
u(t)=\sum_{n=0}^{N-1} a_{n} s\left(t-n T_{c}\right)
$$

where $a_{n}$ are i.i.d., zero-mean, complex-valued random variables and $s(t)$ with support $\left[0, T_{c}\right]$ is the chip waveform. All moments and integrals that appear are assumed to be finite. Using the result in Theorem III.1 we consider $E\left[J_{C}(u)\right]$ and from Equation (5) it suffices to concentrate on $E\left[|\chi(\tau, \nu)|^{2}\right]$. From the independence and zero mean assumptions for the $a_{n} s$ we get the following

$$
\begin{aligned}
E\left[|\chi(\nu, \tau)|^{2}\right]= & {\left[E\left[\left|a_{n}\right|^{2}\right]^{2} \sum_{m=-N+1}^{N-1}(N-|m|) \exp \left(-j 2 \pi m \nu T_{c}\right)\right.} \\
& \left.+N\left(E\left[\left|a_{n}\right|^{4}\right]-E\left[\left|a_{n}\right|^{2}\right]^{2}\right)\right]\left|x_{\varepsilon}(\nu, \tau)\right|^{2} \\
& +E\left[\left|a_{n}\right|^{2}\right]^{2} \sum_{m=1}^{N-1}(N-m)\left(\left|x_{s}\left(\nu, \tau+m T_{c}\right)\right|^{2}+\left|x_{s}\left(\nu, \tau-m T_{c}\right)\right|^{2}\right. \\
= & {\left[E\left[\left|a_{n}\right|^{2}\right]^{2}\left(\frac{\sin \left(\pi \nu T_{c} N\right)}{\sin \left(\pi \nu T_{c}\right)}\right)^{2}+N\left(E\left[\left|a_{n}\right|^{4}\right]-E\left[\left.\left|a_{n}\right|^{2}\right|^{2}\right)\right]\left|x_{s}(\nu, \tau)\right|^{2}\right.} \\
& +E\left[| a _ { n } | ^ { 2 } | ^ { 2 } \sum _ { m = 1 } ^ { N - 1 } ( N - m ) \left(\left|x_{\varepsilon}\left(\nu, \tau+m T_{c}\right)\right|^{2}+\left|x_{s}\left(\nu, \tau-m T_{c}\right)\right|^{2}\right.\right.
\end{aligned}
$$

where $\chi_{s}(\nu, \tau)$ is the ambiguity function of $s(t)$. If $s(t)$ is a rectangular pulse, then

$$
\left|\chi_{s}(\nu, \tau)\right|=\left(T_{c}-|\tau|\right)^{+}\left|\operatorname{sinc}\left[\nu\left(T_{c}-|\tau|\right)\right]\right|,
$$

where $x^{+}=\max (x, 0)$. The general form of the ambiguity function is used to devise simpler upper bounds on the capacity.

Bound on CDMA capacity per unit time

We would like to obtain a bound on the DS-CDMA capacity per unit time. For this we need to let $T$ tend to infinity. For simplicity, take a separable channel, i.e., a channel for which each path fades similarly. Thus, we have

$$
\begin{aligned}
& R_{H}(t, \tau)=R_{H, F}(t) R_{H, T}(\tau), \quad \text { and } \\
& \psi_{H}(\nu, \tau)=\psi_{H, F}(\nu) \psi_{H, T}(\tau) .
\end{aligned}
$$

We also assume that $\int_{t} R_{H, T}(t) d t=1$. The expected rate of the fourthegy is upper bounded by

$$
\begin{aligned}
\lim _{T \rightarrow \infty} \frac{E\left[J_{C}(U)\right]}{T} \leq & {\left[E\left[\left|a_{n}\right|^{2}\right]^{2} \sum_{m=-\infty}^{+\infty}\left|R_{H, F}\left(m T_{c}\right)\right|^{2} T_{c}\right] } \\
& \times \int_{|\tau| \leq T_{c}} \psi_{H, T}(\tau) d \tau \\
& +T_{c}\left(E\left[\left|a_{n}\right|^{4}\right]-E\left[\left|a_{n}\right|^{2}\right]^{2}\right) \int_{\nu} \psi_{H, F}(\nu) d \nu \\
& \times \int_{|r| \leq T_{c}} \psi_{H, T}(\tau) d \tau \\
& +2 T_{c} E\left[\left|a_{n}\right|^{2}\right]^{2} \int_{\nu} \psi_{H, F}(\nu) d \nu \int_{r} \psi_{H, T}(\tau) d \tau .
\end{aligned}
$$

Note that we need $\sum_{m=-\infty}^{+\infty}\left|R_{H, F}\left(m T_{c}\right)\right|^{2}<\infty$ for the bound to be finite. We also need the sum to well-behaved in $T_{c}$. If $S_{H, F}(f)$ is band-limited with bandwidth $F_{d}$, i.e., if there is finite maximum Doppler spread, then by the sampling theorem we have

$$
\sum_{m=-\infty}^{+\infty}\left|R_{H, F}\left(m T_{c}\right)\right|^{2} T_{c}=\int_{\mathbf{t}}\left|R_{H, F}(t)\right|^{2} d t=\int_{f}\left|S_{H, F}(f)\right|^{2} d f
$$

for all $T_{c} \leq \frac{1}{F_{d}}$. Also note that $\int_{\nu} \psi_{H, F}(\nu) d \nu=G_{H}^{2}$. This bound is infinite for the Clarke spectrum. Moreover, we can show that $\frac{E\left[J_{C}[U]\right]}{T} \rightarrow \infty$ in this case. 


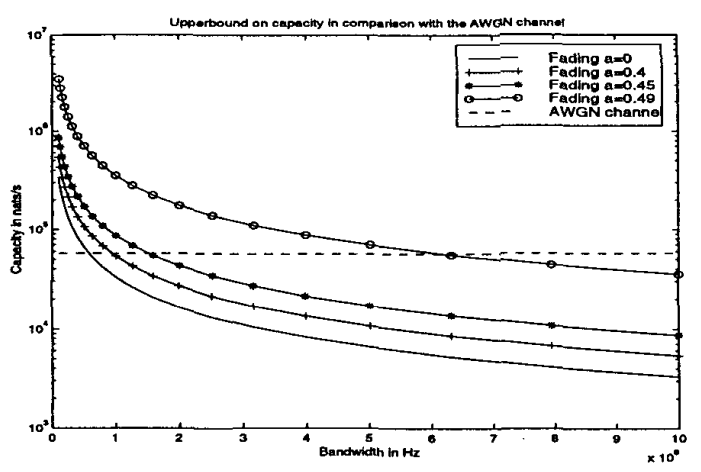

Figure 1: Plot of the upper bound for different values of $a$.

We now plot the bound given in Equation (15) and compare it with the capacity of the AWGN channel. We fix $\frac{P}{N_{0}}$ where $P$ is the receive power, to compare various channels. In terms of our symbols $P=E\left[\left|a_{n}\right|^{2}\right]$ and $N_{0}=\sigma^{2}$. The channel gain is assumed to be 1 . We take $\frac{P}{N_{0}}=1.15 \times 10^{5} \mathrm{~Hz}$. This numerical value is obtained by considering a typical IS-95 CDMA cellular system, corresponding to $\frac{E_{b}}{N_{0}}=6 d B$ and bit rate $28,800 \mathrm{bps}$. We take the distribution accross path delay to be uniform over $\left[0, T_{m}\right.$, where the maximum path delay is taken to be $T_{m}=$ $10^{-6}$ seconds, and the maximum maximum doppler frequency is taken to be $F_{d}=200 \mathrm{~Hz}$. The channel power spectral density for a given path is assumed given by:

$$
S_{H}(f) \propto \frac{1}{\left.\left(1-\left(\frac{F}{F_{d}}\right)^{2}\right)\right)^{a}}, \text { for } 0 \leq a<0.5 .
$$

where $a$ is a parameter with $0 \leq a<0.5$. The well-known Clarke spectrum for uniform angles of arrival in two dimensions, is the limiting case $a=0.5$ (for which case our upper bound is infinite.)

Figure 1 shows the upper bound on the mutual information per unit time as a function of signal bandwidth, for different valudes of $a$. The region of interest is when the upper bound falls below the capacity of an AWGN channel with the same $\frac{P}{N_{0}}$. The figure illustrates that for any $a$ with $0 \leq a<0.5$, the capacity tends to zero as the bandwidth tends to infinity. However, the rate of convergence of the bound it rather slow. For example, if $a=0$ (corresponding to a flat power spectrum for each path delay $\tau$ ), then the capacity upper bound based on fourthegy crosses the upper bound based on energy considerations only for signal bandwidths in excess of $500 \mathrm{MHz}$.

\section{Specular Multi-path channels}

In this subsection we concentrate on specular WSSUS multipath fading channels. Since we are considering a Gaussian channel we only need to specify the correlation function, $R_{H}(t, \tau)$. For an $L$-path specular WSSUS multi-path channel we have the following form for $R_{H}(t, \tau)$,

$$
R_{H}(t, \tau)=\sum_{i=1}^{L} R_{H}^{l}(t) \delta\left(\tau-\tau_{l}\right)
$$

where $\left\{\tau_{1}, \tau_{2}, \ldots, \tau_{L}\right\}$ are the time-offsets of the various multipath components. We can write the following expression for
$J_{C}(u)$

$$
J_{C}(u)=\iint|\chi(\nu, \tau)|^{2} \psi(\nu, \tau) d \tau d \nu=\sum_{l=1}^{L} \sum_{k=1}^{L} J_{C}^{l k}(u),
$$

where

$$
J_{C}^{l k}(u)=\int\left|\chi\left(\nu, \tau_{k}-\tau_{l}\right)\right|^{2} \psi_{H}^{l k}(\nu) d \nu
$$

with

$$
\psi_{H}^{l k}(\nu)=\int S_{H}^{l}(f) S_{H}^{k}(f+\nu) d f
$$

For simplicity consider the case in which the chip waveform is a rectangular pulse and the channel is separable. Then we have that

$$
R_{H}^{l}(t)=p_{l} R_{H}(t) \text { where } \sum_{l=1}^{L} p_{l}=1 .
$$

We can then show that

$$
\begin{aligned}
\lim _{T \rightarrow \infty} \frac{E\left[J_{C}(U)\right]}{T} \leq & E\left[\left|a_{n}\right|^{2}\right]^{2} \sum_{l=1}^{L} p_{l}^{2} \int\left|R_{H}(t)\right|^{2} d t \\
& +\left(E\left[\left|a_{n}\right|^{4}\right]-E\left(\left|a_{n}\right|^{2}\right]^{2}\right) \sum_{l=1}^{L} p_{l}^{2} \int_{-T_{c}}^{T_{C}}\left|R_{H}(t)\right|^{2} d t \\
& +2 E\left[\left|a_{n}\right|^{2}\right]^{2}\left(1-\sum_{l=1}^{L} p_{l}^{2}\right) \int_{-T_{c}}^{T_{c}}\left|R_{H}(t)\right|^{2} d t .(18)
\end{aligned}
$$

Now letting $T_{c}$ tend to 0 , we get

$$
\lim _{T_{c} \rightarrow 0} \lim _{T \rightarrow \infty} \frac{E\left[J_{C}(U)\right]}{T} \leq E\left[\left|a_{n}\right|^{2}\right]^{2} \sum_{l=1}^{L} p_{l}^{2} \int\left|R_{H}(t)\right|^{2} d t .
$$

If we now assume that all paths have equal energy, then $p_{l}=\frac{1}{L}$ and $\sum_{l=1}^{L} p_{l}^{2}=\frac{1}{L}$. Therefore, the capacity per unit time is inversely proportional to the number of paths. Specializing to the case of Telatar and Tse [8] with Gaussian fading and realizing that $E\left[\left|a_{n}\right|^{2}\right]=P$ and $R_{H}(t)=1_{\left[-T_{\text {coherence }}, T_{\text {coherence }}\right]}(t)$ where $T_{\text {coherence }}$ is the coherence time of the channel, we can recover their upper bound on the capacity per unit time for very large spreading factors, namely, $\frac{P^{2} T_{\text {coherence }}}{N_{0}^{2} L}$.

\section{ACKNOWLEDGEMENTS}

The authors are grateful to pointers from Emre Teletar early in this investigation.

\section{REFERENCES}

[1] R. G. Gallager, "Information Theory and Reliable Communication," John Wiley and Sons, New York, 1968.

[2] R. G. Gallager, "Energy Limited Channels: Coding, Multiaccess, and Spread Spectrum," Tech. Report LIDS-P-1714, LIDS, MIT, Cambridge, Mass., 1987, November.

[3] R. Gallager and M. Médard, "Bandwidth scaling for fading channels," Proc. International Symposium on Information Theory'97 (ISIT), Ulm, Germany", 1997, June-July", pp. 471.

[4] R. S. Kennedy, "Fading Dispersive Communication Channels,", Wiley Interscience, New York, 1969.

[5] R. E. Blahut, "Theory of Remote Surveillance," Manuscript to be published by Cambridge University Press.

[6] V. G. Subramanian and B. Hajek, "Capacity and Reliability Function Per Unit Cost for WSSUS Fading Channels," Procetdings of the Conference on Information Systems and Sciences (CISS), Johns Hopkins, 1999.

[7] S. Verdú, "On channel capacity per unit cost," IEEE Trans. Info. Th., 1990, Sept., vol. IT-34, no. 5, pp. 1019-1030.

[8] E. Telatar and D. Tse, "Capacity and Mutual Information of Broadband Multi-path Fading Channels," preprint, 1997. 